\title{
Is UK emergency food nutritionally adequate? A critical evaluation of the nutritional content of UK food bank parcels
}

\author{
L. Turnbull and D. Bhakta \\ Faculty of Life Sciences \& Computing, London Metropolitan University, London, N7 8DB
}

The use of food banks is rising in the UK; a leading charity's records show an increase of $163 \%$ from 2013 and $2014^{(1)}$. Whilst research has examined the causes of food insecurity; to date there has been little investigation into the health implications of widespread and on-going food bank use.

A typical food parcel should consist of food items that will be sufficient for three days depending on whether they are single, a couple, or an entire family and usually consists of a variety of canned foods: soups, beans, tomatoes, vegetables, meats (often as tinned ravioli or spaghetti), fish, fruit and rice pudding. Other goods such as ultra-heat treated (UHT) milk, juice, a bag of sugar, pasta/rice, coffee, cereals and biscuits are also provided ${ }^{(2)}$. However, the variety and content of food parcels can vary depending on the volunteer packing the food parcel, the stock available, population demographics, and by the organisation who runs the food banks. Therefore, we decided to collect information from two different food banks in southwest UK (located in Bournemouth and Blandford). The contents of different food parcels ( $\boldsymbol{n}$ 126) were analysed and typical meal plans were also constructed using available food items to assess actual nutritional adequacy at the point of consumption.

\begin{tabular}{|c|c|c|c|c|}
\hline & $\begin{array}{l}\text { Mean Whole Content } \\
\text { of Parcels }(n \text { 126) }\end{array}$ & $\begin{array}{l}\text { Mean Content of } \\
\text { Meal Plans }\end{array}$ & EAR/ DRVs & $\begin{array}{l}\text { Mean Intake of Low Income } \\
\text { NDNS Quintile }^{(3)}\end{array}$ \\
\hline Energy (kcal) & 2874 & 1547 & 2550 & 1678 \\
\hline Protein (\% intake) & 14 & 17 & 15 & 17 \\
\hline Fat (\% intake) & 19 & 20 & 35 & 34 \\
\hline CHO (\% intake) & 67 & 62 & 50 & 49 \\
\hline
\end{tabular}

Mean energy and \% energy of macronutrient intake of the emergency food parcels met the EAR and DRVs but the constructed meal plans did not meet the requirements for energy and was lower than the mean intake of low income NDNS quintile. Furthermore, both the whole contents and meal plan showed a higher proportion of energy derived from carbohydrate than recommended and the NDNS survey. Similarly, when the meal plans were compared to the EatWell Plate ${ }^{(4)}$ they were deficient in proportions of milk \& dairy foods and fruit \& vegetables groups; mean values of $5 \%$ and $12 \%$ respectively compared to the recommended proportions of $15 \%$ and $32.5 \%$. Micronutrients that were also of possible concern were vitamin $\mathrm{C}$, calcium, magnesium, potassium and zinc, where the meal plans did not meet RNIs, although LRNIs were met. We suggest that reformulation of food bank parcels with the addition of a few low-cost key items could address most of the above issues; one litre of UHT milk per person, add potatoes and pulses (not just baked beans) as a distinct categories. Sugar, fruit juice and pasta/bean/pastry meat items should be counted as "added extras" and not as part of the core parcel, and packet sauce mixes and crackers could be added to increase meal plan options. This should be in addition to providing dietary education, basic cooking skills and instilling a concept of a healthy balanced diet amongst the recipients.

1. Trussell Trust (2014) 'Highlights of the Year 2013-2014'. Available at http://www.trusselltrust.org/resources/documents/Financial-Summary2013-2014-web.pdf (Accessed: 17th March 2015).

2. Trussell Trust (2013-2014) 'Foodbank Information Pack'. Available at http://www.trusselltrust.org/resources/documents/Press/ TT-Foodbank-Information-Pack-2013-14.pdf (Accessed: 5th March 2015).

3. Public Health England and FSA (2014) 'National Diet and Nutrition Survey: results from Years 1 to 4' Available at https://www.gov.uk/government/statistics/national-diet2008-and-2009-to-2011-and-2012 (Accessed: 20th April 2015).

4. Public Health England (2013) 'The EatWell Plate' Available at http://www.nhs.uk/Livewell/Goodfood/Pages/eatwell-plate.aspx (Accessed: 2nd May 2015). 